\title{
Avaliação do peso dos recém-nascidos: o que é normal ou anormal
}

\author{
Evaluation of newborn weight: what is normal and what is abnormal
}

\author{
Marilza Vieira Cunha Rudge
}

O crescimento fetal caracteriza-se por uma seqüência de crescimento de tecidos e órgãos, diferenciação e maturação que são determinados pela oferta materna de substrato, transferência placentária desses substratos e pelo potencial de crescimento determinado pelo genoma. Apesar de vários fatores estarem envolvidos no crescimento fetal, ainda não são bem conhecidos os mecanismos celular e molecular do crescimento normal. Estudos experimentais em ratas diabéticas, modelo de macrossomia e de restrição de crescimento intra-uterino (RCIU), evidenciaram alterações placentárias distintas. Nas placentas de ratas com diabete moderado, modelo de macrossomia fetal, há hiperplasia celular e desaparecimento do glicogênio placentário no final da prenhez. Nas placentas de ratas com diabete grave, modelo de RCIU, a superficie placentária de trocas materno-fetais é mais espessada e, além da hiperplasia, há hipertrofia celular com manutenção dos estoques placentários de glicogênio ${ }^{1}$. Esses resultados evidenciaram que, apesar do aspecto histopatológico semelhante entre placentas de ratas diabéticas, cujos recém-nascidos são macrossômicos ou apresentam RCIU, existem alterações bioquímicas e histoquímicas que explicam os desvios opostos do crescimento fetal. No início da vida fetal o maior determinante do crescimento é o genoma fetal, mas no final da gestação tornam-se importantes o ambiente intra-uterino, a nutrição e a influência hormonal ${ }^{2}$. Os desvios do crescimento fetal para mais ou para menos são importantes, pois estão relacionados com aumento da morbimortalidade perinatal: a macrossomia e a RCIU devem ser diagnosticadas, tratadas e prevenidas. Essa é uma tarefa fundamental da assistência pré-natal de qualidade.

A maior parte do conhecimento atual sobre o crescimento fetal normal e anormal baseia-se em pesos padrões, que são o ponto de referência desse crescimento. O baixo peso ao nascer é definido pela Organização Mundial de Saúde como todo recém-nascido com peso inferior a $2500 \mathrm{~g}$ independente da idade gestacional ${ }^{3}$. A macrossomia é um termo usado, com mais imprecisão, para descrever um feto/ recém-nascido muito grande. Há consenso entre os obstetras que os recém-nascidos com menos de 4000 g não são excessivamente grandes; porém, não há definição sobre a macrossomia. Esse termo macrossomia não aparece no New Shorter Oxford English Dictionary ${ }^{4}$, mas o Stedman's Medical Dictionary ${ }^{5}$ define o "anormalmente de tamanho grande": a palavra chave é "anormal". É dificil estabelecer o limite superior do crescimento humano acima do qual o peso ao nascer é considerado anormal.

A literatura tem mostrado que não apenas o peso ao nascimento é importante, mas também a sua relação com a idade gestacional. Há cerca de 40 anos é que foram elaboradas as primeiras curvas de crescimento fetal usando dados populacionais. Em 1961 foram publicados os valores normais para peso do recém-nascido, comprimento e circunferência craniana utilizados para definir a restrição de crescimento fetal. Em 1966, Gruenwald ${ }^{6}$ relatou que cerca de um terço dos recém-nascidos de baixo peso eram maduros e que seu tamanho pequeno poderia ser explicado pela insuficiência placentária crônica. Esses conceitos, de certa forma recentes, evidenciam que o peso do recém-nascido é determinado não apenas pela idade gestacional, mas também pela taxa de crescimento fetal. Em 1967, Battaglia e Lubchenco ${ }^{7}$ classificaram recém-nascidos em: pequenos para idade gestacional - PIG (peso abaixo do percentil 10), adequados para idade gestacional - AIG (peso entre os percentis 10 e 90) e grandes para idade gestacional - GIG (peso acima do percentil 90). Há inúmeras críticas a esta curva, pois foi construída com os dados de recém-nascidos de mães brancas, hispânicas e que moravam em região de altitude elevada, que são fatores que diminuem o crescimento fetal. Essas críticas levaram à elaboração de várias curvas nacionais e regionais do peso fetal por idade gestacional. No Brasil, foram construídas inúmeras curvas, sendo a de Matheus e Sala ${ }^{8}$ uma das mais conhecidas. O CLAP (Centro Latinoamericano de Perinatologia) ${ }^{9}$ construiu uma curva de peso do recém-nascido em relação à idade gestacional usando mulheres sul-americanas sem patologia. O peso limite de recém-nascidos de termo é cerca de 500 g maior na curva do CLAP comparada à de Lubchenko. (número da citação?)

Entretanto, não apenas o peso deve ser analisado, mas também o padrão de crescimento fetal é de grande valia para detectar os riscos de complicações dos recém-nascidos.

A relação entre o peso ao nascimento e a estatura expressa a realidade do crescimento fetal.

O índice ponderal (IP) de Rohrer foi empregado no trabalho de Santos et al. ${ }^{10}$ para diagnóstico do crescimento intra-uterino restrito. Uma das conclusões do estudo é que o índice de Rohrer diagnosticou

Prof. Titular Obstetrícia - Faculdade de Medicina de Botucatu (SP) - Brasil.

Pró-Reitora de Pós-Graduação da Universidade Estadual de São Paulo - UNESP - Botucatu (SP) - Brasil.

Correspondência

Rua General Telles, 1396 - apto 81 - 18602-120 - Botucatu (SP) - Brasil. 
diferentes padrões de crescimento intra-uterino, que não seriam reconhecidos utilizando-se o peso em função da idade gestacional, mostrando uma acurácia mais elevada para estes diagnósticos. É obtido pela relação entre o peso do recém-nascido em grama x 100/estatura $\left(\mathrm{cm}^{3}\right)$, uma maneira antiga mas coerente de classificação dos recém-nascidos. É uma das várias relações peso/estatura, na qual a lei geométrica da dimensionalidade é mantida: se o volume tridimensional ou se a gravidade são aproximadamente constantes, o peso de um corpo é proporcional ao cubo das dimensões lineares. Quando o índice não é constante, há mudança na forma ou na densidade dos corpos com a mesma idade. Este indice descreve quão pesado é o recém-nascido em relação à estatura e idade. Os números maiores denotam recém-nascido pesado para a sua estatura e os menores descrevem que o mesmo é leve para a sua estatura. A dissociação entre peso e estatura fetais, que é característica da alteração nutricional intra-uterina, pode ser determinada pelo uso do IP de Rohrer. É método de grande valia na estimativa da massa de tecido mole e depósito de gordura fetais, particulares de feto para feto.

A literatura salienta a importância da classificação do peso fetal para os desvios opostos do desenvolvimento fetal (a macrossomia e a RCIU) e da separação dos que são grandes ou pequenos por constituição (simétricos) daqueles com crescimento patológico exagerado ou diminuído (assimétricos). Isto porque o prognóstico perinatal é pior no recém-nascido assimétrico grande ou pequeno $^{6}$. No trabalho de Santos et al. ${ }^{10}$, dos recém-nascidos de baixo peso $(<2500 \mathrm{~g}) 54 \%$ eram assimétricos, porém não foi observado pior prognóstico perinatal nessa população.

Os recém-nascidos PIG com índice ponderal menor que 2,25 constituem a população de RCIU assimétrico, e os com IP entre 2,25 e 3,10, a de RCIU simétrico. A restrição de crescimento com IP menor que 2,25 tem sido descrita como aquele recém-nascido que não atingiu seu potencial máximo de crescimento e pode ser clinicamente reconhecido como portador de deficiência de tecidos moles.

Os macrossômicos assimétricos, com IP maior que 3,10 caracterizam os recém-nascidos GIG assimétricos que apresentam mais complicações neonatais. Isto porque esses fetos têm organomegalia abdominal com conseqüente desproporção entre a circunferência craniana, diâmetro bis-acromial e circunferência abdominal, responsáveis pelos tocotraumatismos, em especial a distócia bis-acromial. O IP de Rohrer usado por Sgarbosa ${ }^{11}$, evidenciou que as gestantes diabéticas e as com hiperglicemia tratadas com dieta ou com dieta e insulina tiveram aumento de recém-nascidos com IP maior que 3.10

O IP, método antigo para a classificação do peso de recém-nascido, é medida a ser implementada nos serviços de perinatologia, pois poderá ser útil na identificação das dificuldades no parto, evitando a distócia de ombro, os traumas esqueléticos e a paralisia braquial dos recém-nascidos de mãe diabética. Tem custo quase zero, é de fácil reprodutibilidade e importante no diagnóstico e condução de recémnascidos a curto, médio e longo prazo diante de diferentes padrões de crescimento intra-uterino ${ }^{12}$.

\section{Referências}

1. Calderon IMP, Rudge MVC, Ramos MD, Peraçoli JC. Estudo longitudinal, bioquímico e histoquímico de placentas de ratas diabéticas: relação com a macrossomia e o retardo de crescimento intra-uterino. Rev Bras Ginecol Obstet. 1999;21(2):91-8.

2. Cunningham FG, Gant N, Leveno KJ, Gilstrap III LC, Aut, JC, Wenstrom KD. Williams obstetrics. 21st ed. New York: McGraw-Hill; 2001.

3. World Health Organization. Public health aspects of low birth weight: third report of the Expert Committee on Maternal and Child Health. Geneva: World Health Organization; 1961. (WHO Technical Reporter Service, 217).

4. Brown L, editor. New shorter Oxford english dictionary. Oxford: Clarendon Press; 1993.

5 Spraycar M, editor. Stedman's medical dictionary. 26th ed. Baltimore: Williams \& Wilkins; 1995. p. 1052.

6. Gruenwald P. Growth of the human fetus. I. Normal growth and its variation. Am J Obstet Gynecol. 1966;94(8):1112-9.

7. Battaglia FC, Lubchenco LO. A practical classification of newborn infants by weight and gestational age. J Pediatr. 1967;71(2):159-63.

8. Matheus M, Sala MA. Relação entre o peso e a estatura neonatal: influência do sexo do recém-nascido. J Bras Ginecol. 1985;95(10):473-6.

9. Fescina R, Schwarcz R, Díaz AG. Vigilância de crescimento fetal: manual de autoinstruções. Montevidéo: Centro Latino Americano de Perinatologia e Desenvolvimento Humano (CLAP); 1992.

10. Santos AMM, Rocha JES, Thomaz ACP. Crescimento intra-uterino diagnosticado pelo índice ponderal de Rohrer e sua associação com morbidade e mortalidade neonatal precoce. Rev Bras Ginecol Obstet. 2005; 27(6):303-9.

11. Sgarbosa F. Índice ponderal: uma maneira antiga e coerente de classificação dos recém-nascidos de mães diabéticas [tese]. Botucatu: Universidade Estadual Paulista "Júlio de Mesquita Filho"; 2003.

12. Caiza Sánchez ME, Diaz Rosselló JL, Simini F. Índice ponderal para calificar a una población de recién nacidos a término. An Pediatr (Barc). 2003;59(1):48-53. 